\title{
Study of Sensitivity of the Algorithm for Assimilating Small Amount of Data in the Ocean Dynamics Model
}

\author{
M. N. Kaurkin ${ }^{1}$, R. A. Ibrayev ${ }^{2,1,3}$ \\ ${ }^{1}$ Shirshov Institute of Oceanology, Russian Academy of Sciences, Moscow, Russian Federation \\ ${ }^{2}$ Marchuk Institute of Numerical Mathematics, Russian Academy of Sciences, Moscow, Russian Federation \\ ${ }^{3}$ Moscow Institute of Physics and Technology (national research university), \\ Dolgoprudny, Russian Federation \\ *kaurkinmn@gmail.com
}

\begin{abstract}
Introduction. The analysis of the original parallel realization of the ensemble optimal interpolation (EnOI) method for data assimilation in the ocean dynamics model developed in the Institute of Numerical Mathematics and the Institute of Oceanology (INMIO model) with a resolution $0.1^{\circ}$ for the North Atlantic region is given in the present paper.

Data and methods. Based on the known ("true") model state of the ocean, the temperature profiles (about 70 per day, up to $1500 \mathrm{~m}$ depth) were chosen and used as synthetic observational data. After the initial condition was perturbed, the numerical experiments were carried out to estimate speed and accuracy of approaching the entire model solution to the "true" state of the ocean as the temperature profiles were assimilated.

Results. Both qualitative analysis of the results and the graphs of the root-mean-square and mean errors of the model solution are given. To study the method sensitivity to the amount of the observational data, the experiments with carried out. They showed that assimilation even of the isolated data could significantly increase the model forecast quality.

Discussion and conclusion. The experiments prove that application of the ensemble optimal interpolation method, even in case very few data, are assimilated in the model, can significantly improve quality both of the model forecast and the entire model solution for those regions where the observational data are very scarce or absent at all. Thus, due to assimilation of the data covering only $3-4$ days, the root-mean-square error for the sea surface temperature model field decreases by $1.5^{\circ} \mathrm{C}$, and the average deviation becomes equal almost to zero over the entire computational domain.
\end{abstract}

Keywords: ocean dynamics modeling, observational data assimilation, ensemble optimal interpolation, eddy-resolving model, Argo data.

Acknowledgments: the study is carried within the framework of the state tasks of FASO, Russia (theme No. 0149-2018-0020) at partial support of RFBR (project No. 16-05-01101). Resources of the Interagency supercomputer center of RAS were used in calculations.

For citation: Kaurkin, M.N. and Ibrayev, R.A., 2019. Study of Sensitivity of the Algorithm for Assimilating Small Amount of Data in the Ocean Dynamics Model. Physical Oceanography, [ejournal] 26(2), pp. 96-103. doi:10.22449/1573-160X-2019-2-96-103

DOI: $10.22449 / 1573-160 X-2019-2-96-103$

(C) 2019, M. N. Kaurkin, R. A. Ibrayev

(C) 2019, Physical Oceanography

\section{Introduction}

In the present paper, the research on the ocean state forecast of the using ensemble assimilation methods is continued. There won't be any review of the works on assimilation, as it was given in our previous publications in sufficient detail. In [1], a parallel implementation of the Ensemble Optimal Interpolation 
(EnOI) method was presented for assimilating observational data in an eddyresolving model of the World Ocean. The effectiveness of the parallel method developed was studied using temperature and salinity data from Argo [2] drifters and $A V I S O$ satellite altimetry data [3]. These works resulted in the confirmation that the assimilation of data by the forecasted three-dimensional fields of temperature and salinity even in those regions where the data were not available. Consequently, perhaps, from the variety of data on observations of the ocean state, their minimum number can be chosen, so that the forecast accuracy is maximum.

Surprisingly, even an excess amount of data currently exists for the restoration and forecast of mesoscale ocean dynamics. First of all, it concerns satellite data (ocean surface temperature and altimetry). On their basis, methods are developed for measuring salinity and currents of the ocean surface, near-surface wind, etc. The state of the upper two-kilometer ocean layer is monitored by Argo buoys. A large amount of information comes from ocean gauges fixed in space. For example, in [4], a methodology for joint processing of small hydrological and satellite altimetry observations is presented. It makes possible to restore the threedimensional temperature and salinity fields very successfully. All this information is used in systems for the ocean state forecast at different time scales (see the reviews in [5-7]). The work [8] is also worth to be reviewed. There the numerical experiments are carried out based on the joint ECHAM-HOPE model and the ensemble assimilation method, and then the results of the perturbations of the model fields and the propagation of these perturbations are analyzed. The study [9] discusses methods for calculating the dynamics of the ocean and the Black Sea using the hydrological observational data assimilation based on the application of the Kalman filter and the principles of adaptation of hydrophysical model fields.

The present papers is aimed to validate a parallel mastering algorithm for the INMIO ocean dynamics model. An attempt to estimate of the sensitivity of the forecast to the initial state and some variations in the assimilated data amount is going to be carried out. Obviously, studies on the dependence of forecast accuracy on the number, type and geographical distribution of ocean data are important for optimizing observational systems. The features of the parallel ensemble optimal interpolation method are also to be denoted.

\section{Ocean Model and Parallel Ensemble Optimal Interpolation Method}

To carry out numerical experiments, the INMIO ocean dynamics model will be applied. It was developed to study the circulation of the World Ocean waters and its individual waters in a wide range of spatial and temporal scales. A more detailed description of the model and numerical experiments on its verification with the $0.5^{\circ}-0.1^{\circ}$ resolution is given in $[10,11]$. Along with the ocean model, the CMF3.0 Compact Modeling Framework will be used to solve the problem of joint modeling of the Earth system and its high-resolution components on computers with distributed memory. The CMF3.0 platform is applied for interprocessor exchanges inside model components, interpolating data between various model grids of components and working with the file system (data input/output) [12].

The ensemble optimal interpolation method EnOI is described in detail in $[13,14]$. It is currently used for the operational forecast of the ocean state 
in a number of systems (see, for example, [15-17]). A detailed description of the parallel method EnOI developed and used in this study is given in [1].

\section{Numerical Experiments}

Algorithm validation. To test the system operation consisting of the ocean model and the assimilation algorithms, numerical experiments were carried out for the North Atlantic region. The model was integrated from 01.01.1948 to 01.05.2008, every 10 days the full state of the model (control points) was maintained. Thus, when assimilating data for May 2008, three control points were used for each May during 1985-1998 as elements of the ensemble for the EnOI method. A total of 40 elements in the ensemble, the cut-off radius is not used, because it is not so important in this method [18]. The conditions of the experiments are the same as in $[2,3]$. The only difference is the use of synthetic observational data obtained based on the known ocean state model, which will be consider "true".

Based on the A01 control experiment, few $Y_{S O D}$ synthetic observation data were obtained, distributed over the domain as the Argo data (about 70 profiles per day, up to $1500 \mathrm{~m}$ depth). Synthetic data were located with the same periodicity and randomness as the Argo measurement data (not necessarily in the model grid nodes). To project data to these points from the model grid (that is, to build the $H$ projection matrix), bilinear interpolation was used. Then, two experiments T01 and T02 were carried out with a perturbed initial condition (corresponding to the model solution for another year), but the atmospheric effect was used in May 2008. However, in the T02 experiment, $Y_{S O D}$ data will be assimilated daily (see table).

\section{Description of the experiments}

\begin{tabular}{c|r|l}
\hline Experiment & $\begin{array}{c}\text { Date of the initial } \\
\text { condition }\end{array}$ & \multicolumn{1}{c}{ Use of observational data $Y_{S O D}$} \\
\hline$A 01$ & 01.05 .2008 & Daily record of $Y_{S O D}$ \\
$T 01$ & 01.05 .2000 & No application \\
$T 02$ & 01.05 .2000 & Daily assimilation of $Y_{S O D}$ by the EnOI method \\
\hline
\end{tabular}

N o t e. COREII atmospheric forcing for 01.05-30.05.2008 is used

In Fig. 1, showing the model sea surface temperature (SST) for various experiments, the location of the $Y_{S O D}$ data is demonstrated to get an idea of their number. The size of the circles in Fig. $1 a$ and 1c is proportional to the difference between the temperature at the point of observation and at the model one. A cross in a circle means that the model temperature is below the profile temperature, a point in the circle - that it's higher. For ease of comparison, in Fig. $1 b$, the asterisks mark the $Y_{S O D}$ data that is generated in the A01 experiment. On the fragments, two areas are circled, which the effect from the assimilation of YSOD data is the most noticeable in. It is worth noting that there were no observations in the Gulf of Mexico throughout the experiment, but the SST in it was significantly adjusted due to the assimilation. 

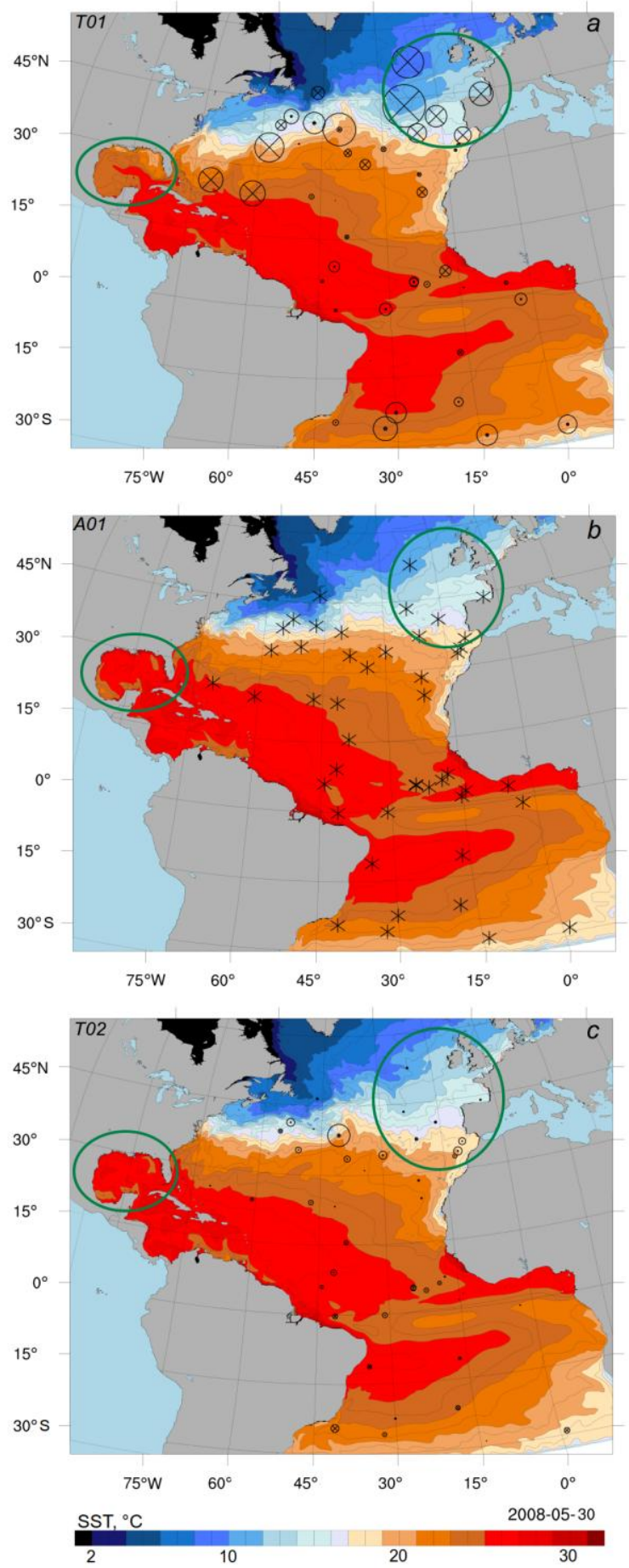

F i g. 1. Sea surface temperature (SST) in the North Atlantic Ocean model on 30.05.2008 in the A01 "true" experiment $(b)$ and also in the T01 $(a)$ and T02 (c) experiments with the perturbed initial condition 
Fig. 2 shows the difference between the SST model fields in the T01, T02 experiments and in the A01 "true" experiment for May 30, 2008 (model date). Comparing Fig. $2 a$ and $2 b$, it is clearly seen how the assimilation corrects the solution in the entire computational domain, and not only at the points where the $Y_{S O D}$ data are located. The effect of data assimilation in the area of the Gulf Stream is especially pronounced.
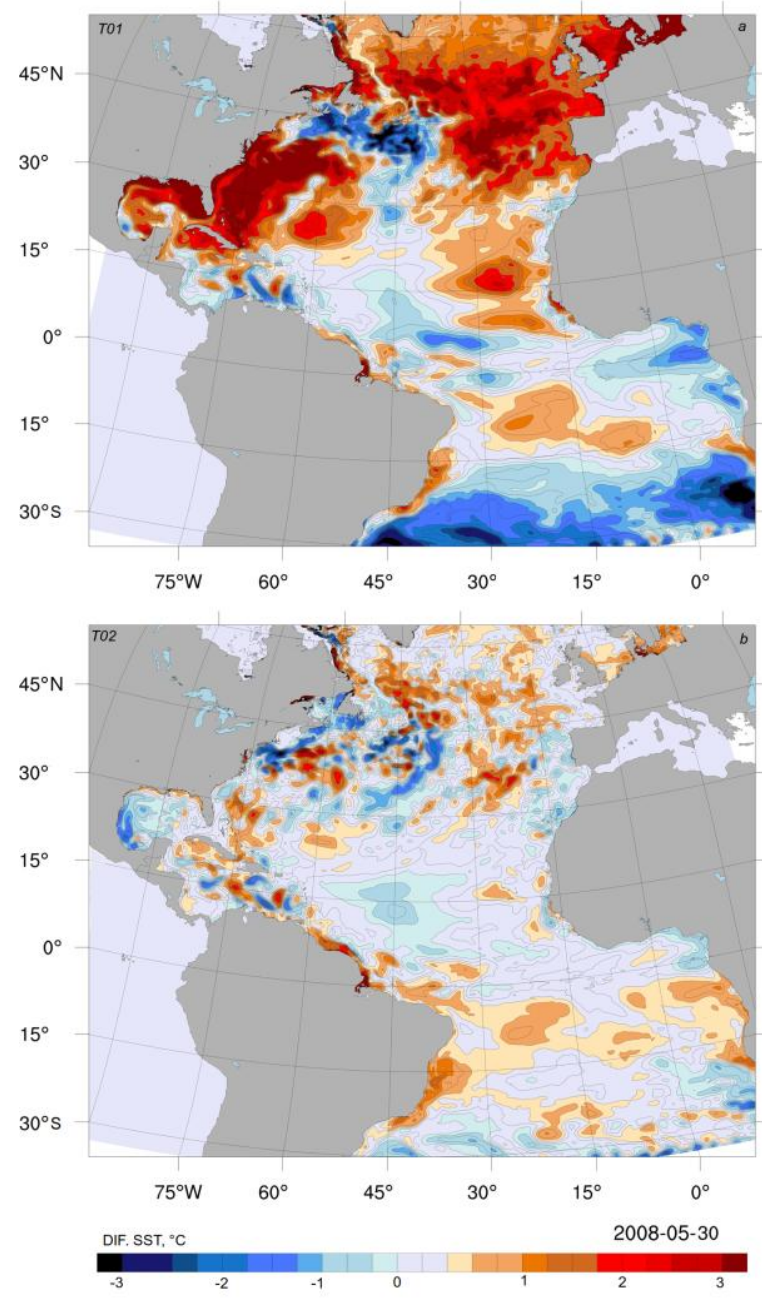

F i g. 2. Difference between the sea surface temperature (SST) model fields on 30.05.2008: $\mathrm{SST}_{A 01}-$ $\mathrm{SST}_{T 01}(a) ; \mathrm{SST}_{\mathrm{AOI}}-\mathrm{SST}_{T 02}(b)$

Graphs of the root-mean-square and average errors of SST calculated over all the computational nodes of the model grid are given in Fig. 3. They show that the $Y_{S O D}$ assimilation greatly approximates the SST model field in the T02 experiment to the model field in the A01 experiment over the entire computational domain. This confirms the correct operation of the implemented data assimilation method, the use of which with the application of $Y_{S O D}$ small observational data (located with the same density as the Argo data) can bring all the model solution to the "true" ocean state (A01experiment). 


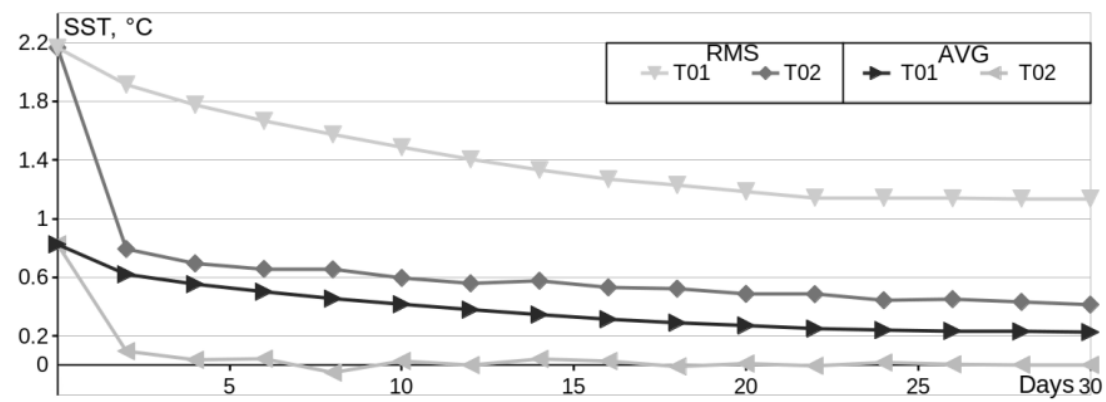

F i g. 3. Root-mean-square (RMS) and average (AVG) errors in the $\mathrm{T} 01$ and $\mathrm{T} 02$ experiments in comparison to the SST "true" field from the A01 Experiment

Study of the method sensitivity to the amount of data used. For this purpose, in addition to the T02 experiment, similar experiments were carried out on the synthetic $Y_{S O D}$ data assimilation, but with a decreasing number of observation points. In addition to the T02 experiment, a total of 4 experiments were carried out with $1 / 2,1 / 4,1 / 8$ and $1 / 16$ of all temperature profiles. In the experiment with 1/16 of the data, only 4-5 profiles per day were assimilated. Graphs of the root-meansquare and average errors of the SST model field for these experiments are shown in Fig. 4. From the analysis of this figure, it can concluded that the method applied is highly efficient with few observational data, so that even the assimilation of single data can significantly improve the quality of modeling.
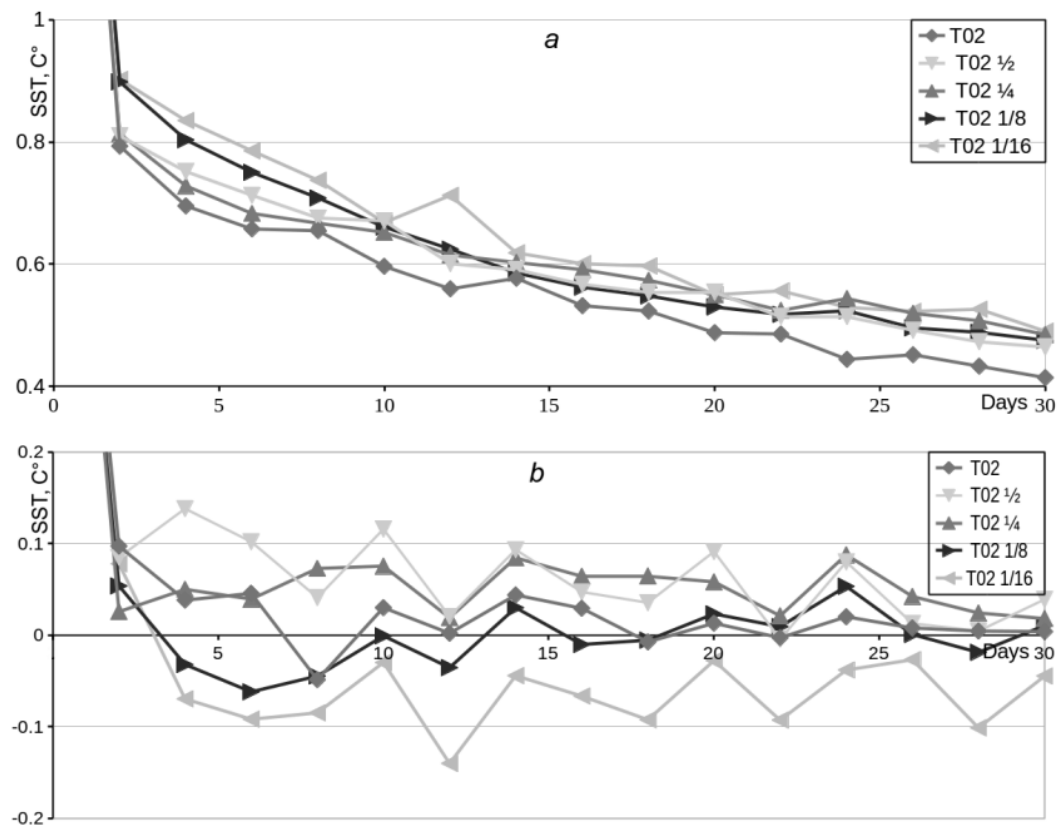

F i g. 4. Root-mean square (RMS) $(a)$ and average (AVG) $(b)$ errors of the sea surface temperature (SST) model field calculated in all the mesh nodes in the experiments based on T02 with assimilation of all the temperature profiles, and 1/2,1/4,1/8 and 1/16 parts of them in comparison to the sea surface temperature (SST) "true" field in the A01 Experiment 


\section{Conclusions}

The present article continues the study of the previously proposed parallel method of assimilating observational data based on the ensemble optimal interpolation EnOI using the example of the INMIO ocean dynamics model of high resolution for the North Atlantic region. Numerical experiments with the use of small synthetic observation data were carried out. A feature of this approach before using field measurement data (satellite or drift one) is the ability to estimate the speed at which a model solution approaches the "true" ocean state, which is unknown in field experiments. It is shown that the use of the EnOI method with the involvement of relatively small data can significantly improve the entire model solution even in those regions where there are no observational data. Only due to the assimilation of small observational data in just 3-4 days, the root-meansquare error for the model field of temperature decreases by $1.5^{\circ} \mathrm{C}$, and the average deviation becomes almost zero over the entire computational domain.

\section{REFERENCES}

1. Kaurkin, M., Ibrayev, R. and Koromyslov, A., 2016. EnOI-Based Data Assimilation Technology for Satellite Observations and ARGO Float Measurements in a High Resolution Global Ocean Model Using the CMF Platform. In: V. Voevodin and S. Sobolev, eds., 2016. Supercomputing. RuSCDays 2016. Communications in Computer and Information Science, vol. 687. Cham: Springer, pp. 57-66. https://doi.org/10.1007/978-3-319-55669-7_5

2. Kaurkin, M.N., Ibrayev, R.A. and Belyaev, K.P., 2016. ARGO Data Assimilation into the Ocean Dynamics Model with High Spatial Resolution Using Ensemble Optimal Interpolation (EnOI). Oceanology, [e-journal] 56(6), pp. 774-781. https://doi.org/10.1134/S0001437016060059

3. Kaurkin, M.N., Ibrayev, R.A. and Belyaev, K.P., 2018. Assimilation of the AVISO Altimetry Data into the Ocean Dynamics Model with a High Spatial Resolution Using Ensemble Optimal Interpolation (EnOI). Izvestiya, Atmospheric and Oceanic Physics, [e-journal] 54(1), pp. 56-64. https://doi.org/10.1134/S0001433818010073

4. Korotaev, G.K., Lishaev, P.N. and Knysh, V.V., 2016. Reconstruction of the ThreeDimensional Salinity and Temperature Fields of the Black Sea on the Basis of Satellite Altimetry Measurements. Izvestiya, Atmospheric and Oceanic Physics, [e-journal] 52(9), pp. 961-973. https://doi.org/10.1134/S0001433816090152

5. Schiller, A. and Brassington, G.B., eds., 2011. Operational Oceanography in the 21st Century. Dordrecht, the Netherlands: Springer, 745 p. doi:10.1007/978-94-007-0332-2

6. Oke, P.R., Larnicol, G., Fujii, Y., Smith, G.C., Lea, D.J., Guinehut, S., Remy, E., Alonso Balmaseda, M., Rykova, T., Surcel-Colan, D., Martin, M.J., Sellar, A.A., Mulet, S. and Turpin, V., 2015. Assessing the Impact of Observations on Ocean Forecasts and Reanalyses: Part 1, Global Studies. Journal of Operational Oceanography, [e-journal] 8(sup1), pp. s49s62. https://doi.org/10.1080/1755876X.2015.1022067

7. Cummings, J., Bertino, L., Brasseur, P., Fukumori, I., Kamachi, M., Martin, M.J., Mogensen, K., Oke, P., Testut, C.E., Verron, J. and Weaver, A., 2009. Ocean Data Assimilation Systems for GODAE. Oceanography, [e-journal] 22(3), pp. 96-109. https://doi.org/10.5670/oceanog.2009.69

8. Belyaev, K.P., Tuchkova, N.P. and Cubash, U., 2010. Response of a Coupled Ocean-IceAtmosphere Model to Data Assimilation in the Tropical Zone of the Pacific Ocean. Oceanology, [e-journal] 50(3), pp. 306-316. https://doi.org/10.1134/S0001437010030021

9. Knysh, V.V., Korotaev, G.K., Mizyuk, A.I. and Sarkisyan, A.S., 2012. Assimilation of Hydrological Observation Data for Calculating Currents in Seas and Oceans. Izvestiya, Atmospheric and Oceanic Physics, [e-journal] 48(1), pp. 57-73. https://doi.org/10.1134/S0001433812010057 
10. Ibrayev, R.A., Khabeev, R.N. and Ushakov, K.V., 2012. Eddy-Resolving $1 / 10^{\circ}$ Model of the World Ocean. Izvestiya, Atmospheric and Oceanic Physics, [e-journal] 48(1), pp. 37-46. https://doi.org/10.1134/S0001433812010045

11. Ushakov, K.V., Ibrayev, R.A. and Kalmykov, V.V., 2015. Simulation of the World Ocean Climate with a Massively Parallel Numerical Model. Izvestiya, Atmospheric and Oceanic Physics, [e-journal] 51(4), pp. 362-380. https://doi.org/10.1134/S0001433815040131

12. Kalmykov, V.V., Ibrayev, R.A., Kaurkin, M.N. and Ushakov, K.V., 2018. Compact Modeling Framework v3.0 for High-Resolution Global Ocean-Ice-Atmosphere Models. Geoscientific Model Development, [e-journal] 11(10), pp. 3983-3997. https://doi.org/10.5194/gmd-113983-2018

13. Evensen, G., 2003. The Ensemble Kalman Filter: Theoretical Formulation and Practical Implementation. Ocean Dynamics, [e-journal] 53(4), pp. 343-367. https://doi.org/10.1007/s10236-003-0036-9

14. Evensen, G. Data Assimilation: The Ensemble Kalman Filter. Berlin: Springer, 2009. 307 p. https://doi.org/10.1007/978-3-642-03711-5

15. Tanajura, C.A.S., Santana, A.N., Mignac, D., Lima, L.N., Belyaev, K. and Ji-Ping, X., 2014. The REMO Ocean Data Assimilation System into HYCOM (RODAS_H): General Description and Preliminary Results. Atmospheric and Oceanic Science Letters, [e-journal] 7(5), pp. 464-470. doi:10.3878/j.issn.1674-2834.14.0011

16. Oke, P.R., Brassington, G.B., Griffin, D.A. and Schiller, A., 2010. Ocean Data Assimilation: A Case for Ensemble Optimal Interpolation. Australian Meteorological and Oceanographic Journal, [e-journal] 59, pp. 67-76. doi:10.22499/2.5901.008

17. Cummings, J.A., 2005. Operational Multivariate Ocean Data Assimilation. Quarterly Journal of the Royal Meteorological Society, [e-journal] 131(613), pp. 3583-3604. https://doi.org/10.1256/qj.05.105

18. Oke, P.R., Sakov, P. and Corney, S.P., 2007. Impacts of Localization in the EnKF and EnOI: Experiments with a Small Model. Ocean Dynamics, [e-journal] 57(1), pp. 32-45. https://doi.org/10.1007/s10236-006-0088-8

\section{About the authors:}

Maxim N. Kaurkin - Research Associate, IO RAS (36, Nakhimovskiy Ave., Moscow, 117997, Russian Federation), Ph.D. (Phys.-Math.), ResearcherID: S-1416-2016, kaurkinmn@ gmail.com

Rashit A. Ibrayev - Chief Research Associate, INM RAS (8, Gubkin St., Moscow, 119333, Russian Federation), Corresponding Member of RAS, Dr.Sci. (Phys.-Math.), ResearcherID: S-6750-2016, ibrayev@mail.ru

Contribution of the co-authors:

Rashit A. Ibrayev - scientific supervision, formulation of the research problem, critical analysis and revision of the text

Maxim N. Kaurkin - software implementation of algorithms, formulation and carrying out the computational experiments and their analysis and the text preparation

All the authors have read and approved the final manuscript.

The authors declare that they have no conflict of interest. 